\title{
Online learning algorithms for wireless energy harvesting nodes
}

\author{
Maria Gregori and Jesús Gómez-Vilardebò \\ Centre Tecnològic de Telecomunicacions de Catalunya (CTTC) \\ \{maria.gregori, jesus.gomez\}@cttc.cat
}

\begin{abstract}
Energy harvesting has emerged as an appealing technology to recharge battery powered devices. Recently, an extensive research has been conducted on the design of power allocation policies for energy harvesting devices. Most works have focused on offline policies that assume non-causal knowledge of the energy harvesting process. Only a few works have considered online policies with the more realistic assumption of only having past knowledge of the energy harvesting process; however, these works generally incur an additional assumption on the knowledge of the probability distribution of the harvested energy (e.g. Poisson distribution) leading to online algorithms that are rarely applicable with available energy harvesting technologies. This paper proposes three online power allocation algorithms capable of learning from the harvested energy in previous days and that perform, in average, as well as the best fixed offline strategy. The numerical results validate the performance of the proposed algorithms when energy is harvested through solar panels.
\end{abstract}

\section{INTRODUCTION}

Energy harvesting is broadly known as the process of converting available ambient energy (e.g., solar or wind energy) into usable electric energy and is envisioned as a promising technology to power wireless nodes. By using energy harvesters, wireless nodes can operate almost perpetually without the need of tedious battery replacements. However, current technologies can only provide limited power levels, e.g., a solar panel harvests around $10 \mathrm{~mW} / \mathrm{cm}^{2}$ [1]. In this context, the design of efficient power allocation algorithms is of paramount importance.

Power allocation algorithms can be classified as: (i) offline algorithms that assume full knowledge of the harvested energy and Channel State Information (CSI) (i.e., about the past and future); and (ii) online algorithms that only consider causal knowledge of these parameters. Offline power allocation strategies have been widely studied in recent years as they provide bounds on the best achievable performance [2]-[6]. However, they can be rarely used in practice due to need of predicting the CSI and harvested energy. In contrast, only a few works have considered online algorithms [6][8]. Existing online algorithms generally capitalize on Markov decision processes and dynamic programming [6]. The main inconvenience of these approaches are that: (i) they require a substantial computational burden, becoming unpractical for low-power devices; and (ii) they assume statistical knowledge of the energy harvesting process. For example, energy arrivals

This work is partially supported by the Spanish Government through the projects INTENSYV (TEC2013-44591-P) and E-CROPs (PCIN-2013-027) in the framework of ERA-NET CHIST-ERA, and by the Catalan Government (2014 SGR 1567).

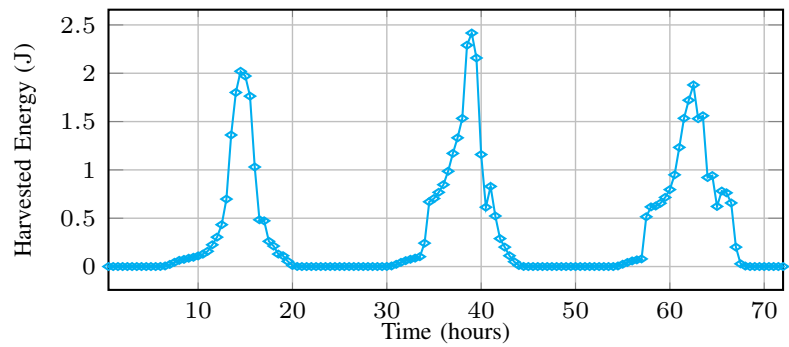

Fig. 1. Harvested energy in three consecutive days. Each day is divided in $N=48$ slots of duration 30 minutes. The harvested energy is taken from the real traces in [9] for a solar panel of $100 \mathrm{~cm}^{2}$ and $10 \%$ conversion efficiency.

are generally modeled as being independent and identically distributed following a Poisson distribution. This is unrealistic for certain energy harvesters, e.g., solar panels, for which the harvested energy clearly depends on the time of the day as shown in Fig. 1 [9], [10]. These two facts, obviously limit the applicability of existing online algorithms.

A different approach explores online policies that perform well in the worst case energy harvesting profile and channel fading, in terms of the competitive rate ratio [11] (ratio between offline and online performances) and competitive rate gap [12], [13] (difference between offline and online performances), respectively.

In opposition to the aforementioned works, we use the framework of online convex optimization [14] to derive online algorithms capable of learning the optimal policy from observing the previous days. We propose three low-complexity online algorithms whose average asymptotic performance is as good as the one of the best fixed offline policy, which knows the harvested energy and CSI in advance. This is confirmed by the numerical results under different energy harvesting profiles, including realistic energy harvesting profiles from solar panels.

\section{INTRODUCTION TO ONLINE CONVEX OPTIMIZATION}

In this section, we briefly introduce the framework of Online Convex Optimization (OCO) (see [14]). The protocol of OCO can be understood as a repeated game between an online player and its opponent, which can be nature. At every round $t=$ $1, \ldots, T$, the player selects an action $\mathrm{x}^{t}$ from the action set $\mathcal{X}$. Then, the opponent makes its move by selecting a cost function $f^{t} \in \mathcal{F}: \mathcal{X} \rightarrow \mathbb{R}$, where $\mathcal{F}$ is the bounded set of cost functions available to the opponent. In OCO, the player aims at designing an algorithm, $\mathcal{A}$, that at each round $t$ takes an action $\mathrm{x}^{t}$ based on the previous actions and the observed costs. The efficiency of the sequence generated by the algorithm, $\left\{\mathbf{x}^{t}\right\}_{t=1}^{T}$, is characterized relative to a comparator sequence 
$\left\{\overline{\mathbf{x}}^{t}\right\}_{t=1}^{T}$ in terms of regret, which measures the difference on the total cost of the two sequences,

$$
\operatorname{Regret}_{T}\left(\mathcal{A},\left\{\overline{\mathbf{x}}^{t}\right\}_{t=1}^{T}\right)=\sum_{t=1}^{T} f^{t}\left(\mathbf{x}^{t}\right)-f^{t}\left(\overline{\mathbf{x}}^{t}\right) .
$$

The goal is to design an algorithm, $\mathcal{A}$, that achieves a sublinear regret as a function of $T$, i.e., Regret $_{T}=o(T)$. This implies that the average regret, Regret $_{T} / T$, tends to zero as $T$ goes to infinity. In other words, it implies that the online policy, $\left\{\mathbf{x}^{t}\right\}_{t=1}^{T}$, performs in average as well as the comparator sequence $\left\{\overline{\mathbf{x}}^{t}\right\}_{t=1}^{T}$.

A broadly used comparator is the batch policy that uses the best static action given the opponent moves in advance and is obtained as $\overline{\mathbf{x}}_{s}^{t}=\arg \min _{\mathbf{x}} \sum_{t=1}^{T} f^{t}(\mathbf{x}), \forall t$. Only very recently, [15] has considered online learning algorithms that operate in dynamic environments. In that case, the comparator sequence becomes also dynamic and is $\overline{\mathbf{x}}_{d}^{t}=\arg \min _{\mathbf{x}} f^{t}(\mathbf{x})$. For any algorithm $\mathcal{A}$, the dynamic regret is necessarily greater or equal to the static regret, $\operatorname{Regret}_{T}\left(\mathcal{A},\left\{\overline{\mathbf{x}}_{d}^{t}\right\}_{t=1}^{T}\right) \geq$ $\operatorname{Regret}_{T}\left(\mathcal{A},\left\{\overline{\mathbf{x}}_{s}^{t}\right\}_{t=1}^{T}\right)$.

\section{SYSTEM MODEL AND PROBLEM FORMULATION}

We consider a point to point communication between a transmitter powered by energy harvesting and its intended receiver. The transmission time, e.g., a day, is composed of $N$ time slots of duration $T_{s}$. It is assumed that the channel gain, $h_{n} \in\left[0, h_{\text {Max }}\right]$ remains constant within each slot, $n=$ $1, \ldots, N$. At the beginning of each slot the transmitter harvests a packet of energy $e_{n} \in\left[0, e_{\text {Max }}\right]$. This energy is stored in a rechargeable battery until it is used for transmission. We assume that the battery has no leakage and infinity capacity. In this context, the transmitter aims at designing the transmission powers $\left\{p_{n}\right\}_{n=1}^{N}$ that maximize the total daily sum-rate and satisfy the Energy Causality Constraints (ECCs). Assuming Gaussian signaling, this problem reads as follows

$$
\begin{aligned}
\max _{\left\{p_{n}\right\}_{n=1}^{N}} & \sum_{n=1}^{N} \log \left(1+h_{n} p_{n}\right) \\
\text { subject to } & T_{s} \sum_{n=1}^{\ell} p_{n} \leq \sum_{j=1}^{\ell} e_{j}, \quad \ell=1, \ldots, N,
\end{aligned}
$$

where the ECCs in (1b) impose that the cumulatively expended energy cannot be greater than the cumulative harvested energy.

The problem in (1) has been widely studied in an offline setup by assuming that the transmitter knows in advance the values of future channel gains and harvested energies. The optimal power allocation of (1) is the well-known Directional Water-Filling (DWF) solution [6].

In the following lines, we explore online power allocation algorithms that do not require future knowledge of the channel state and energy harvesting. The proposed iterative algorithms consist of $T$ iterations (see Fig. 2), where at each iteration $t=$ $1, \ldots, T$, the algorithms propose an online power allocation for the next $N$ time slots, aimed at solving an instance of the problem in (1).

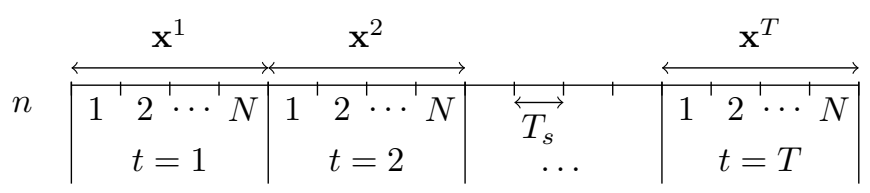

Fig. 2. Temporal representation for designing an online power allocation algorithm. $T$ stands for total number of iterations and each iteration is composed of $N$ time slots.

Remark 1 (Time scale selection). As represented in Fig. 2 there are two temporal indexes of interest $n=1, \ldots, N$ and $t=1, \ldots, T$. Each round $t$ is composed of $N$ slots. Thus, $T$ denotes the total time horizon for which we want to run the online power allocation algorithm, which could be perpetually, i.e., $T \rightarrow \infty$; whereas, $N$ denotes the length in time slots of the power allocation policy. In particular, the time duration $N T_{s}$ must be carefully selected to promote the learning capabilities of the online algorithms, exploiting the existing temporal correlation of the harvested energy and channel gain (if any). For example, if the node harvests solar energy and we want to allow power adjustments every half an hour, $T_{s}=30$ minutes, then we would set $N=48$ allowing the power allocation $\left\{p_{n}\right\}_{n=1}^{N}$ to span a whole day. In this context, $t$ would stand for the day index. This allows the algorithms proposed in the following to learn the distribution of the energy harvesting and channel state information, improving their behavior day after day.

As shown next, the proposed algorithms are able to learn the behavior of the batch power allocation and asymptotically achieve the same average sum-rate. First, we rewrite the problem in (1) to fall within the OCO framework as follows

$$
\min _{\mathbf{x} \in \mathcal{X}}-\sum_{n=1}^{N} \log \left(1+\frac{h_{n}}{T_{s}} \sum_{j=1}^{n} x_{j}(n) e_{j}\right),
$$

where $x_{j}(n)$ stands for the fraction of energy harvested at the $j$-th slot that is consumed at the $n$-th slot. Let $\mathbf{x} \triangleq\left(\mathbf{x}_{n}\right)_{n=1}^{N}$ with $\mathbf{x}_{n} \triangleq\left(x_{j}(n)\right)_{j=1}^{n}$. Since energy can only be consumed after being harvested, we have $n \geq j$. Note that the $N$-th inequality constraint in (1b) is necessarily met with equality as the rate increases with power. Accordingly, we can impose the energy causality constraints in (1b) by means of the feasible set $\mathcal{X}=\prod_{j=1}^{N} \mathcal{X}_{j}$ with $\mathcal{X}_{j}=\left\{x_{j}(n) \geq 0: \sum_{n=j}^{N} x_{j}(n)=\right.$ $1\}$. Observe that by increasing the number of optimization variables from $N$ in (1) to $X \triangleq \sum_{n=1}^{N} n=N(N+1) / 2$ in (2) and defining the feasible set $\mathcal{X}$, we have been able to formulate the problem without constraints.

Now, the problem in (2) falls withing the framework of OCO. At each round, $t$, the player selects and action $\mathrm{x}^{t} \in \mathcal{X}$ and the opponent (in this case nature) selects the parameters $h_{n}^{t}$ and $e_{j}^{t}, \forall n, j=1, \ldots, N$. The opponent's choice determines the cost function $f^{t}(\mathbf{x})=\sum_{n=1}^{N} f_{n}^{t}(\mathbf{x})$ with $f_{n}^{t}\left(\mathbf{x}_{n}\right)=$ $-\log \left(1+\frac{h_{n}^{t}}{T_{s}} \sum_{j=1}^{n} x_{j}(n) e_{j}^{t}\right)$.

\section{ONLINE POWER ALLOCATION ALGORITHMS}

In this section we propose three different online power allocation algorithms for wireless energy harvesting nodes and theoretically bound their regret. We fo- 


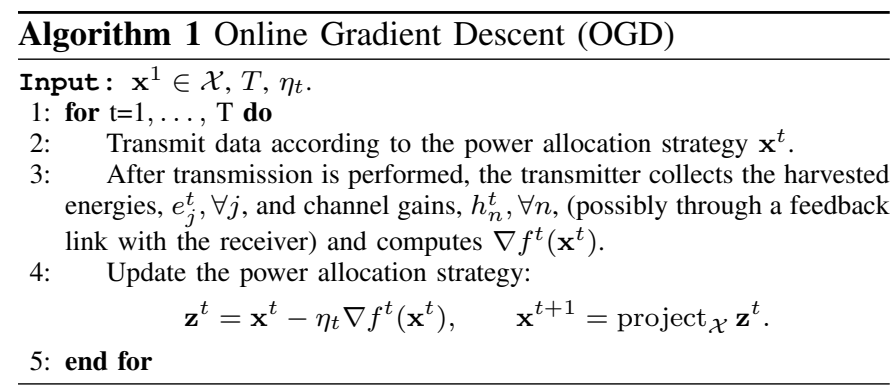

cus on characterizing the regret with respect to the static comparator (or batch policy) $\overline{\mathbf{x}}_{s}$, i.e., $\overline{\mathbf{x}}_{s}=$ $\arg \min _{\mathbf{x} \in \mathcal{X}}-\sum_{t=1}^{T} \sum_{n=1}^{N} \log \left(1+\frac{h_{n}^{t}}{T_{s}} \sum_{j=1}^{n} x_{j}(n) e_{j}^{t}\right)$. The regret of the dynamic comparator, which corresponds to the DWF solution, will be solely evaluated through numerical simulations in Section V. Generally, the performance of online algorithms is evaluated with respect to three main metrics: (i) the worst case regret; (ii) convergence speed; and (iii) the computational complexity. As shown in the following lines, there is an inherent tradeoff between these metrics.

\section{A. Online gradient descent}

The Online Gradient Descent (OGD) algorithm is presented in Algorithm 1. Note that the algorithm does not require CSI nor energy harvesting information to determine the power allocation strategy at each iteration. However, after finishing the $t$-th iteration, the transmitter uses the collected information of the harvested energy and channel gains (possibly through a feedback link with the receiver) to perform the update of the power allocation strategy. This update is performed by moving towards the steepest descent direction of the cost function at the current point, $-\nabla f^{t}\left(\mathrm{x}^{t}\right)$. This step entails a computational complexity of $O(X)$ per iteration (recall that $X=N(N+1) / 2)$. Additionally, a projection into the feasible set is needed to ensure that the new iterate is feasible, where project $_{\mathcal{X}} \mathbf{z}^{t}=\operatorname{argmin}_{\mathbf{x} \in \mathcal{X}}\left\|\mathbf{x}-\mathbf{z}^{t}\right\|_{2}$. Since the set $\mathcal{X}$ is the Cartesian product of $N$ unit simplexes, the projection over $\mathcal{X}$ can be efficiently computed by using the algorithm proposed in [16], leading to a complexity of $O\left(N^{2} \log N\right)$. Accordingly, the complexity of the OGD is $O\left(N^{2} \log N\right)$ per iteration.

In the following lemma, we show that the regret of the OGD algorithm is sublinear in $T$.

Lemma 1. The OGD algorithm with step sizes $\eta_{t}=$ $2 Q /\left(\gamma_{\operatorname{Max}} \sqrt{(N+1) t}\right)$ guarantees the following regret bound for all $T>1$ :

$$
\operatorname{Regret}_{T}\left(O G D, \overline{\mathbf{x}}_{s}\right) \leq \frac{1}{2} \gamma_{M a x} N \sqrt{(N+1) T}(2 Q+1 / Q)
$$

where $\gamma_{\text {Max }} \triangleq h_{M a x} e_{M a x} / T_{s}$ denotes the maximum signal to noise ratio and $Q>0$ is a constant.

Proof: From [14, Theorem 3.1] and using $\eta_{t}>\eta_{t+1}$, $t=1 \cdots T-1$, we have $\operatorname{Regret}_{T}\left(O G D, \overline{\mathbf{x}}_{s}\right) \leq 1 / 2\left(D^{2} / \eta_{T}+\right.$ $\left.G^{2} \sum_{t=1}^{T} \eta_{t}\right)$, where $D$ is the diameter of set $\mathcal{X}$, and $G$ is the maximum Lipschitz constant of the costs functions associated to the $\ell_{2}$ norm. The proof of the lemma follows by particularizing $\eta_{t}, D$, and $G$, which are obtained in Lemmas 4 and

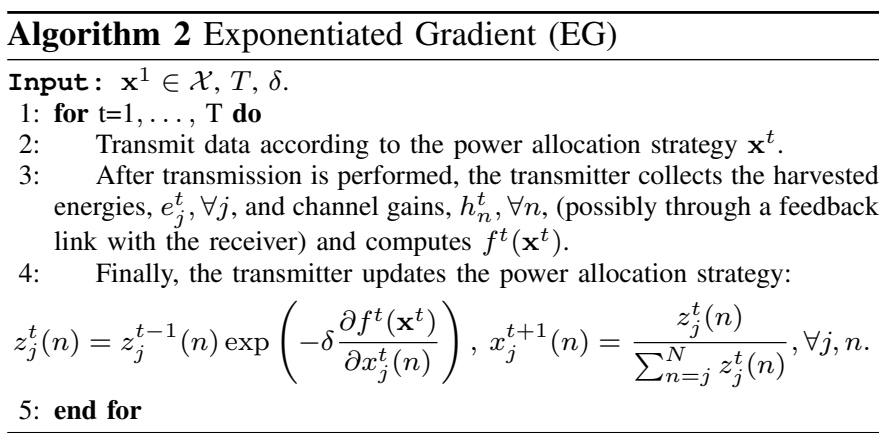

5 , respectively. The proof also uses that $\sum_{t=1}^{T} 1 / \sqrt{t} \leq 2 \sqrt{T}$ [14].

It can be easily verified that, for this step size $\eta_{t}$, the constant $Q$ that achieves the lowest regret bound is $Q^{\star}=1 / \sqrt{2}$. Nevertheless, as shown in the numerical results, by selecting $Q>Q^{\star}$ faster convergence rates can be achieved at a cost of increasing the regret bound. Finally, observe that, for any $Q>0$, the average regret tends to zero as $T$ goes to infinity, $\lim _{T \rightarrow \infty}$ Regret $_{T}\left(O G D, \overline{\mathbf{x}}_{s}\right) / T=0$.

\section{B. Exponentiated gradient}

Online mirror descent algorithms [14] are an important subclass of online algorithms. In these algorithms, the update is performed in a dual (or mirror) space, where duality is defined in terms of a regularization. This regularization serves as a transformation of the space in which the gradient updates are performed.

In Algorithm 2, we present the online mirror descent algorithm with the negative entropic regularization, which leads to the so-called Exponentiated Gradient (EG) algorithm [14]. The structure of the feasible set $\mathcal{X}$, which is the Cartesian product of unit simplexes, motivates the use of the negative entropic regularization since it transforms the projection in $\mathcal{X}$ to a simple scaling by the $\ell_{1}$ norm. As a result, the power allocation update in Step 4 of Algorithm 2 is obtained in closed form. The complexity of this step is $O\left(X^{2}\right)$ per iteration.

Next, we show that the EG achieves a tighter regret bound than the OGD.

Lemma 2. The EG algorithm with step size $\delta=$ $W \sqrt{N \log ((N+1) / 2) /\left(2 T \gamma_{\text {Max }^{2}}\right)}$ guarantees the following regret bound for all $T>1$ :

$\operatorname{Regret}_{T}\left(E G, \overline{\mathbf{x}}_{s}\right) \leq \gamma_{\text {Max }} \sqrt{2 T N \log \left(\frac{N+1}{2}\right)}(W+1 / W)$ where $W>0$ is a constant.

Proof: From Theorem 5.1, Lemma 5.4, and Corollary 5.5 of [14], we have $\operatorname{Regret}_{T}\left(E G, \overline{\mathbf{x}}_{s}\right) \leq D_{R} / \delta+2 \delta G_{\infty}^{2} T$ where $D_{R}$ is the diameter of set $\mathcal{X}$ given the entropic regularization, and $G_{\infty}$ is the maximum Lipschitz constant of the costs functions associated to the $\ell_{\infty}$ norm. Using the step size in the lemma we have $\operatorname{Regret}_{T}\left(E G, \overline{\mathbf{x}}_{s}\right) \leq$ $\sqrt{D_{R} 2 T G_{\infty}^{2}}(W+1 / W)$. The proof follows by particularizing the values of $G_{\infty}$ and $D_{R}$, which are obtained in Lemmas 5 and 6, respectively (see the Appendix). Additionally, we have 


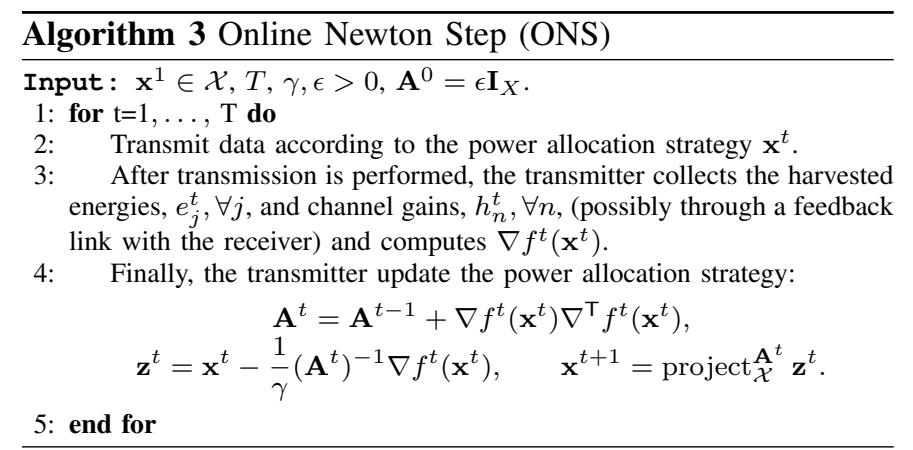

used that the entropic regularization is 1-strongly convex with respect to the $\ell_{1}$ norm, whose dual norm is the $\ell_{\infty}$ norm.

Again, we can further optimize the step size over the constant $W$, obtaining $W^{\star}=1$. Nevertheless, as for the OGD, in practice one might prefer to select $W>W^{\star}$ to speed up the convergence of the algorithm. Finally, the EG algorithm performs in average as well as the static comparator, i.e., $\lim _{T \rightarrow \infty} \operatorname{Regret}_{T}\left(E G, \overline{\mathbf{x}}_{s}\right) / T=0, \forall W>0$.

\section{Online Newton step}

The Online Newton Step (ONS) algorithm, described in Algorithm 3, is able to provide tighter regret bounds as function of $T$ than the OGD and EG algorithms by exploiting the exp-concavity of the functions $f^{t}$ (see the Appendix). The ONS was originally proposed in [17] and similarly to the classical Newton method [18] exploits the curvature of the objective function to compute the new update. This is done by multiplying by the inverse of the matrix $\mathbf{A}^{t}$ in the update step, which is related to the Hessian of $f^{t}$ due to its exp-concavity.

Note that in comparison with the OGD the ONS requires an additional $O\left(X^{2}\right)$ storage to store the matrix $\mathbf{A}^{t}$ and the computation of the inverse of this matrix at every iteration. However, the inverse can be computed (if invertible) by using the matrix inversion lemma with a complexity $O\left(X^{2}\right)$. Additionally, the ONS requires the projection of $\mathbf{z}^{t}$ according to the norm induced by the matrix $\mathbf{A}^{t}$, i.e., project $\mathbf{A}_{\mathcal{X}} \mathbf{z}^{t}=$ $\operatorname{argmin}_{\mathbf{x} \in \mathcal{X}}\left(\mathbf{x}-\mathbf{z}^{t}\right)^{\top} \mathbf{A}^{t}\left(\mathbf{x}-\mathbf{z}^{t}\right)$. This problem can be solved in polynomial time up to a certain accuracy [17] and represents the major source of complexity of the ONS algorithm. To avoid this complexity the use of a modulo call to a projection oracle was suggested in [17].

Next, we derive the regret of the ONS algorithm.

Lemma 3. The ONS algorithm with $\gamma=$ $1 /(2 R) \min \left\{\left(4 \gamma_{\text {Max }} N \sqrt{N+1}\right)^{-1}, 1\right\}$ and $\epsilon=(\gamma \sqrt{2 N} R)^{-2}$ guarantees the following regret bound for all $T>4$

$$
\begin{aligned}
& \operatorname{Regret}_{T}\left(O N S, \overline{\mathbf{x}}_{s}\right) \leq \\
& 5 R\left(1+\gamma_{\text {Max }} N \sqrt{N+1}\right) \frac{N(N+1)}{2} \log (T)
\end{aligned}
$$

where $R \geq 1$ is a constant.

Proof: To obtain this result, we have particularized the values of $G$ and $D$ given in Lemma 5 and 4, respectively, in [14, Theorem 4.3]. Second, we have added the constant $R$ in the step size.

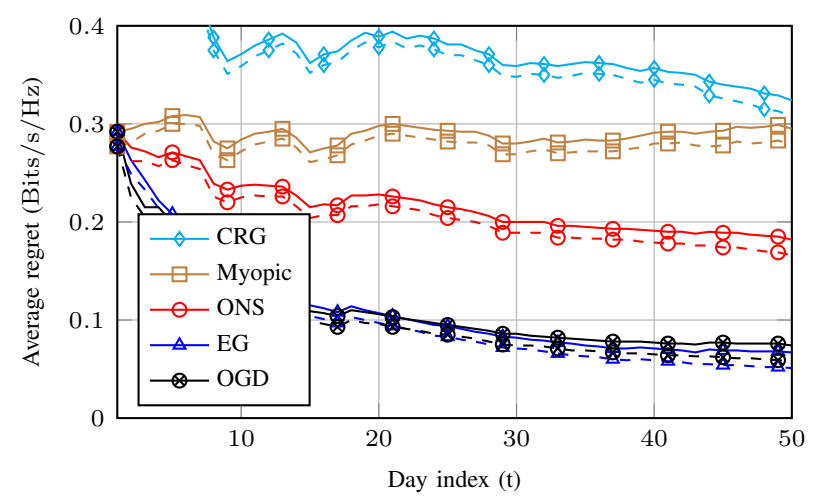

Fig. 3. Average regret of the different algorithms and comparators. The regret with respect to the dynamic and static comparators is depicted with the solid and dashed lines, respectively.

\section{NUMERICAL RESULTS}

This section numerically evaluates the regret of the proposed algorithms in realistic energy harvesting scenarios. The proposed algorithms are evaluated with respect to: (i) the myopic policy, which in each time slot distributes the harvested energy evenly among the remaining slots, namely $x_{j}(n)=(N-j+1)^{-1}$. This policy is optimal in terms of competitive rate ratio [11]. (ii) the Competitive Rate Gap (CRG) policy proposed in [12], [13] that for static channels achieves a constant rate gap. These policies are designed to perform close to the offline strategy in the worst scenario, but cannot learn the statistics of the energy harvesting and fading processes. Unless otherwise specified, we consider a node equipped with a solar panel of dimension $100 \mathrm{~cm}^{2}$ and $10 \%$ efficiency. The harvested energy is taken from real traces obtained from the data repository in [9], where we have divided the day in $N=48$ time slots of duration 30 minutes, i.e., $T_{s}=1800 \mathrm{~s}$ (an example is shown in Fig. 1). We consider a static channel, whose channel gain has been obtained considering a central frequency of $2.4 \mathrm{GHz}$, a bandwidth of $B=30 \mathrm{MHz}$, an equivalent noise temperature of $290 \mathrm{~K}$ with a noise factor of 10 , and the path loss is obtained using the Friis transmission equation with unit antenna gains and a distance of $50 \mathrm{~m}$ between transmitter and receiver. The proposed algorithms are initialized with the myopic policy.

In this setup, Fig. 3 depicts the average regret, $\operatorname{Regret}_{t}\left(\mathcal{A},\left\{\overline{\mathbf{x}}^{\tau}\right\}_{\tau=1}^{t}\right) /(N t)$, of the different algorithms with respect to the static and dynamic comparators, where $t$ is the day index that varies in the $x$ axis. For this simulation, we have set $T=50$ and the step-size parameters to $Q=W=R=50$. For each algorithm, the regret with respect to the dynamic comparator (the offline optimal DWF solution) is depicted with the solid lines; whereas, the regret with respect to the static comparator is shown in dashed lines. It is observed that for the different algorithms, the two regrets (static and dynamic) follow a similar trend. As expected, the static regret is always smaller or equal to the dynamic one. Additionally, the gap between the two slightly increases with the number of iterations. It is important to remark here that the static and dynamic comparators are only required to evaluate the obtained regret, but its computation is not necessary for the 


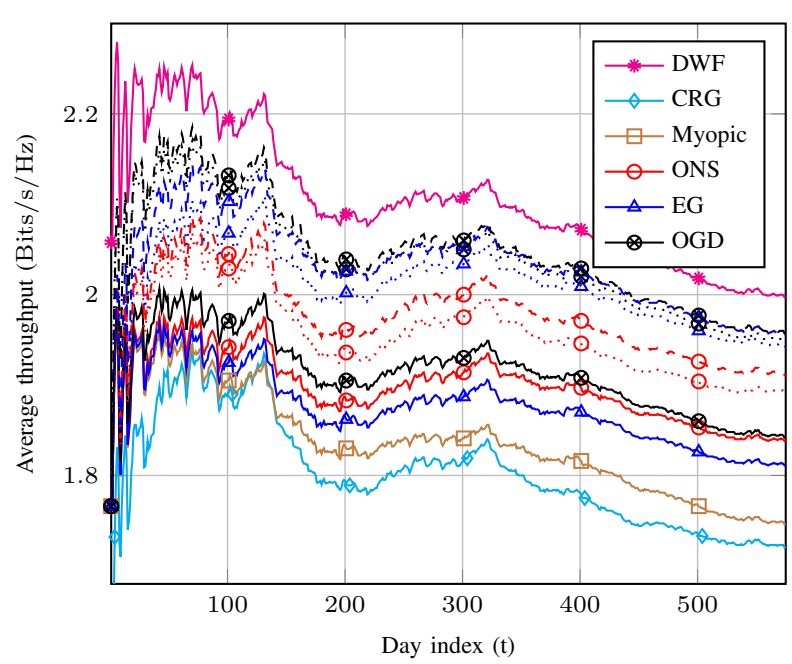

Fig. 4. Average throughput for different values of $Q, W$, and $R$. The solid lines stand for $Q=W=R=1$, the dotted lines for $Q=W=R=25$, and the dashed ones for $Q=W=R=50$.

proposed algorithms. In this context, note that the computational complexity of the static comparator increases with $T$. Thus, the computation of the static comparator becomes intractable when $T$ grows. To cope with this, in the subsequent simulations, we will only evaluate the regret of the dynamic comparator; this regret upper bounds the regret of the static comparator as argued in Section II.

In Figs. 4 and 5, we evaluate the effect of varying the parameters $Q, W$, and $R$, which affect the step size and regret of the proposed algorithms. Fig. 4 depicts the average throughput, $\sum_{\tau=1}^{t} f^{\tau}\left(\mathbf{x}^{\tau}\right) /(N t)$. In contrast to the myopic policy, the proposed online algorithms, OGD, EG, and ONS, progressively approach the optimal offline DWF solution (the dynamic comparator). Interestingly, by increasing the parameters $Q, W$, and $R$ the algorithms approach much faster to the optimal solution. This behaviour is more clearly observed when looking at the average regret in Fig. 5 (recall that it is the difference between the offline optimal DWF solution and online policies). We want to point out that after 500 days and until the end of the simulation at $T=575$, the maximum loss with respect to the offline optimal DWF solution, $\overline{\mathbf{x}}_{d}^{t}$, defined as, $100\left(f^{t}\left(\mathbf{x}^{t}\right)-f^{t}\left(\overline{\mathbf{x}}_{d}^{t}\right)\right) / f^{t}\left(\overline{\mathbf{x}}_{d}^{t}\right)$, is $4.7506 \%, 4.14 \%$, and $7.44 \%$ for the OGD, EG, and ONS algorithms, respectively; whereas, the minimum relative loss is as low as $0.52 \%, 0.16 \%$, and $0.77 \%$. Finally, we want to remark that the theoretical bounds for the worst case regret derived in Lemmas 1, 2, and 3 are much larger than the regrets obtained in practical scenarios.

Finally, in Fig. 6, we evaluate the proposed online algorithms for a different energy harvesting distribution. In particular, we consider that the harvested energy packets in each time slot are independent and identically distributed from a uniform distribution, where the maximum and minimum values are the same as in the previous simulations. In contrast to the solar energy harvesting simulations, now the CRG outperforms the Myopic policy. However, as before, the proposed algorithms are able to learn the energy harvesting and fading processes,

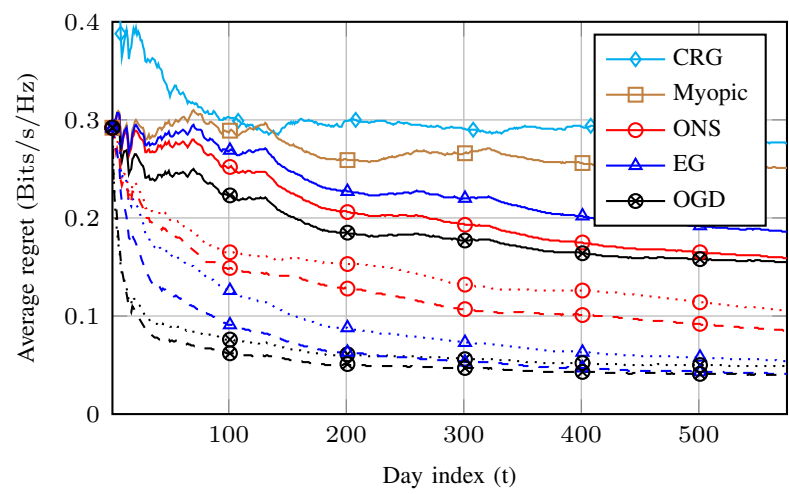

Fig. 5. Average regret for different values of $Q, W$, and $R$. The solid lines stand for $Q=W=R=1$, the dotted lines for $Q=W=R=25$, and the dashed ones for $Q=W=R=50$.

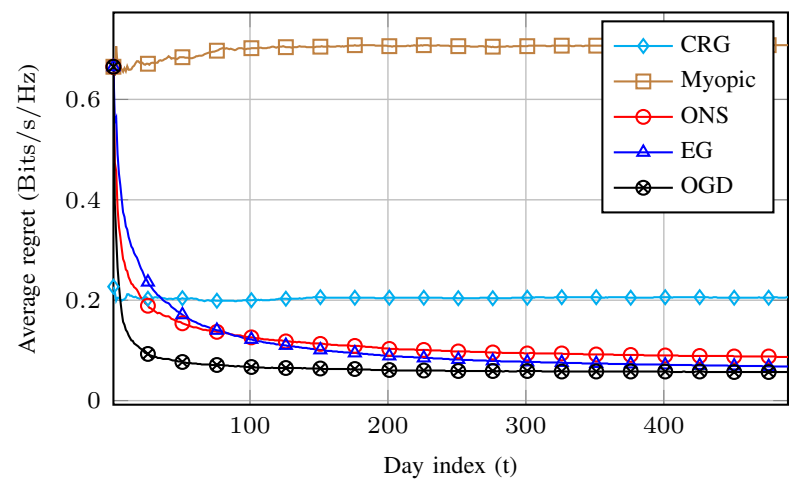

Fig. 6. Average regret for $Q=W=R=50$ and a uniform distribution of the harvested energy.

reducing the regret day after day.

\section{CONCLUSIONS}

This paper has proposed three different online power allocation algorithms that are able to adapt and approach the optimal offline power allocation policy. It has been shown that the worst case (static) regret of the proposed algorithms is sublinear with respect to the time horizon, implying that the average regret of the algorithms tends to zero as the time horizon goes to infinity. The numerical results have shown that the proposed algorithms are able to learn the optimal power allocation policy independently of the distribution of the harvested energy. When comparing the different algorithms, we have seen that the ONS algorithm achieves the smallest worse case regret bound; however, it has the highest complexity due to the projection step. In contrast, the OGD and EG algorithms have a lower computational complexity but higher regret bounds. However, in the numerical experiments, these algorithms present faster convergence rates than the ONS algorithm. We have also seen that both the OGD and ONS algorithms are horizon free, which is an important feature for the perpetual operation of wireless energy harvesting nodes. To conclude, we believe that the final choice of the algorithm will depend on the specific use case and requirements of the node; however, we strongly believe that the OGD is a good candidate since it is horizon free and simple, while still has a good performance. As future research lines, we will theoretically 
study the regret with respect to the dynamic comparator, and we will include additional constraints in the problem such as finite battery capacity and mask constraints on the transmission power.

\section{APPENDIX}

In the following lemmas, we characterize the structure of the feasible set $\mathcal{X}$ and the function $f^{t}$.

Lemma 4. The maximum diameter of the set $\mathcal{X}$ is upper bounded by $D=\sqrt{2 N}$, i.e., $\forall \mathbf{x}, \mathbf{y} \in \mathcal{X},\|\mathbf{x}-\mathbf{y}\|_{2} \leq D=$ $\sqrt{2 N}$.

$$
\text { Proof: }\|\mathbf{x}-\mathbf{y}\|_{2}^{2} \leq \sum_{j=1}^{N} \sum_{n=j}^{N} x_{j}(n)^{2}+y_{j}(n)^{2} \leq
$$
$\sum_{j=1}^{N} \sum_{n=j}^{N} x_{j}(n)+y_{j}(n)=\sum_{j=1}^{N} 2=2 N$.

Lemma 5. $f^{t}(\mathbf{x})$ is a Lipschitz continuous function on $\mathcal{X}$ and satisfies:

(a) $\forall \mathbf{x}, \mathbf{y} \in \mathcal{X},\left|f^{t}(\mathbf{x})-f^{t}(\mathbf{y})\right| \leq G\|\mathbf{x}-\mathbf{y}\|_{2}$

(b) $\forall \mathbf{x}, \mathbf{y} \in \mathcal{X},\left|f^{t}(\mathbf{x})-f^{t}(\mathbf{y})\right| \leq G_{\infty}\|\mathbf{x}-\mathbf{y}\|_{\infty}$,

with $G=\gamma_{\operatorname{Max}} \sqrt{N(N+1) / 2}$ and $G_{\infty}=\gamma_{\text {Max }}$.

Proof: The following inequalities hold: $\left|f^{t}(\mathbf{x})-f^{t}(\mathbf{y})\right| \stackrel{(i)}{\leq}$

$\left|f^{t}(\mathbf{x})-\left(f^{t}(\mathbf{x})+\nabla_{x} f^{t}(\mathbf{x})^{\top}(\mathbf{y}-\mathbf{x})\right)\right| \stackrel{(i i)}{\leq}\left\|\nabla_{\mathbf{x}} f^{t}(\mathbf{x})\right\| \| \mathbf{x}-$ $\mathbf{y} \|$, where in (i) we have used that a convex function is lower bounded by its linearization at any point; and (ii) uses the Cauchy-Schwartz inequality. Next we show that $\left\|\nabla_{\mathbf{x}} f^{t}(\mathbf{x})\right\|_{2}^{2} \leq G^{2}$. Note that $\nabla_{\mathbf{x}} f^{t}(\mathbf{x})=$ $\left[\nabla_{\mathbf{x}_{1}} f_{1}^{t}\left(\mathbf{x}_{1}\right) \ldots \nabla_{\mathbf{x}_{N}} f_{N}^{t}\left(\mathbf{x}_{N}\right)\right]^{\top}$, where the $j$-th component of $\nabla_{\mathbf{x}_{n}} f_{n}^{t}\left(\mathbf{x}_{n}\right)$ is $\frac{\partial f_{n}^{t}\left(\mathbf{x}_{n}\right)}{\partial x_{j}(n)}=\frac{-h_{n}^{t} e_{j}^{t} / T_{s}}{1+\frac{h_{n}^{n}}{T_{s}} \sum_{q=1}^{n} x_{q}(n) e_{q}^{t}}, j \leq n$. Thus, $\left\|\nabla_{\mathbf{x}} f^{t}(\mathbf{x})\right\|_{2}^{2} \leq \sum_{n=1}^{N} \sum_{j=1}^{n}\left(h_{n}^{t} e_{j}^{t} / T_{s}\right)^{2} \leq G^{2}$.

Finally, the proof of b) follows from the definition of the $\ell_{\infty} \operatorname{norm}\left\|\nabla_{\mathbf{x}} f^{t}(\mathbf{x})\right\|_{\infty}=\max _{n, j \leq n}\left|\frac{-h_{n}^{t} e_{j}^{t} T_{s}^{-1}}{1+\frac{h_{n}^{t}}{T_{s}} \sum_{q=1}^{n} x_{q}(n) e_{q}^{t}}\right| \leq$ $\gamma_{\text {Max }}$.

Lemma 6. Given the vector $\mathrm{x} \in \mathcal{X}$, the negative entropic regularization is $R(\mathbf{x})=\sum_{n=1}^{N} \sum_{j=1}^{n} x_{j}(n) \log \left(x_{j}(n)\right)$. The diameter of the set $\mathcal{X}$ with respect to the regularization $R(\mathbf{x})$ is $D_{R}=\max _{\mathbf{x}, \mathbf{y} \in \mathcal{X}} R(\mathbf{y})-R(\mathbf{x}) \leq N \log \left(\frac{N+1}{2}\right)$.

Proof: Since $x_{j}(n) \in[0,1]$, we have $R(\mathbf{x}) \leq 0, \forall \mathbf{x} \in \mathcal{X}$, where the equality is attained when only one component of $x_{j}(n)$ is one and all the other ones are zero. Thus, $\max _{\mathbf{y} \in \mathcal{X}} R(\mathbf{y})=0$. Note that $-x \log x$ is a concave function, then by Jensen's inequality we have

$$
\begin{aligned}
-\sum_{n=1}^{N} \sum_{j=1}^{n} & \frac{x_{j}(n) \log \left(x_{j}(n)\right)}{X} \leq \\
& -\left(\frac{\sum_{n=1}^{N} \sum_{j=1}^{n} x_{j}(n)}{X}\right) \log \left(\frac{\sum_{n=1}^{N} \sum_{j=1}^{n} x_{j}(n)}{X}\right)
\end{aligned}
$$

where $X=\sum_{n=1}^{N} \sum_{j=1}^{n} 1=N(N+1) / 2$. By using this, it readily follows that $\max _{\mathbf{x} \in \mathcal{X}}-R(\mathbf{x}) \leq N \log ((N+1) / 2)$. Thus, $D_{R} \leq N \log \left(\frac{N+1}{2}\right)$.

Lemma 7. The function $f^{t}$ is $\alpha$-exp-concave, with $\alpha=1$.
Proof: The function $f^{t}$ is $\alpha$-exp-concave if and only if $\nabla^{2} f^{t}(\mathbf{x}) \succeq \alpha \nabla_{\mathbf{x}} f^{t}(\mathbf{x}) \nabla_{\mathbf{x}} f^{t}(\mathbf{x})^{\top}$ [14]. Let $\mathbf{a}_{n}^{t} \triangleq\left(\frac{h_{n}^{t}}{T_{s}} e_{j}^{t}\right)_{j=1}^{n}$, then $f_{n}^{t}\left(\mathbf{x}_{n}\right)=-\log \left(1+\mathbf{x}_{n}^{\top} \mathbf{a}_{n}^{t}\right)$. Thus, we have $\nabla_{\mathbf{x}_{n}} f_{n}^{t}\left(\mathbf{x}_{n}\right)=-\mathbf{a}_{n}^{t} /\left(1+\mathbf{x}_{n}^{\top} \mathbf{a}_{n}^{t}\right)$ and $\nabla_{\mathbf{x}_{n}}^{2} f_{n}^{t}\left(\mathbf{x}_{n}\right)=\mathbf{a}_{n}^{t}\left(\mathbf{a}_{n}^{t}\right)^{\top} /\left(1+\mathbf{x}_{n}^{\top} \mathbf{a}_{n}^{t}\right)^{2}$. Accordingly, $\nabla_{\mathbf{x}_{n}}^{2} f_{n}^{t}\left(\mathbf{x}_{n}\right) \succeq \nabla_{\mathbf{x}_{n}} f_{n}^{t}\left(\mathbf{x}_{n}\right) \nabla_{\mathbf{x}_{n}} f_{n}^{t}\left(\mathbf{x}_{n}\right)^{\mathrm{T}}$. This proves that $f_{n}^{t}\left(\mathbf{x}_{n}\right)$ is $\alpha$-exp-concave for any $\alpha \leq 1$. Finally, note that $\nabla_{\mathbf{x}}^{2} f^{t}(\mathbf{x})$ is a block diagonal matrix, where the diagonal matrices are $\nabla_{\mathbf{x}_{n}}^{2} f_{n}^{t}\left(\mathbf{x}_{n}\right), n=1, \ldots, N$. Thus, $f^{t}(\mathbf{x})$ is also $\alpha$-exp-concave for any $\alpha \leq 1$.

\section{REFERENCES}

[1] R. J. Vullers, R. Schaijk, H. J. Visser, J. Penders, and C. V. Hoof, "Energy harvesting for autonomous wireless sensor networks," IEEE J. Solid-State Circuits, vol. 2, no. 2, pp. 29-38, Spring 2010.

[2] M. Gregori and M. Payaró, "Energy-efficient transmission for wireless energy harvesting nodes," IEEE Trans. Wireless Commun., vol. 12, no. 3 , pp. 1244-1254, Mar. 2013.

[3] _ " "On the precoder design of a wireless energy harvesting node in linear vector Gaussian channels with arbitrary input distribution," IEEE Trans. Commun., vol. 61, no. 5, pp. 1868-1879, May 2013.

[4] — , "On the optimal resource allocation for a wireless energy harvesting node considering the circuitry power consumption," IEEE Trans. Wireless Commun., vol. 13, no. 11, pp. 5968-5984, Nov 2014.

[5] _ - "Optimal power allocation for a wireless multi-antenna energy harvesting node with arbitrary input distribution," in Proceedings of the IEEE International Conference on Communications, Jun. 2012, pp. 5794 -5798 .

[6] O. Ozel, K. Tutuncuoglu, J. Yang, S. Ulukus, and A. Yener, "Transmission with energy harvesting nodes in fading wireless channels: Optimal policies," IEEE J. Sel. Areas Commun., vol. 29, no. 8, pp. 1732-1743, Sep. 2011.

[7] W. Zeng, Y. Zheng, and C. Xiao, "Online precoding for energy harvesting transmitter with finite-alphabet inputs and statistical CSI," IEEE Trans. Veh. Technol., vol. PP, no. 99, pp. 1-1, 2015.

[8] J. Rubio, O. Muñoz-Medina, and A. Pascual-Iserte, "A stochastic approach for resource allocation with backhaul and energy harvesting constraints," IEEE Transactions on Vehicular Technology, 2015.

[9] M. Gorlatova, M. Zapas, E. Xu, M. Bahlke, I. J. Kymissis, and G. Zussman, "CRAWDAD dataset columbia/enhants (v. 2011-04-07)," Downloaded from http://crawdad.org/columbia/enhants/20110407, Apr. 2011.

[10] M. Miozzo, D. Zordan, P. Dini, and M. Rossi, "Solarstat: Modeling photovoltaic sources through stochastic markov processes," in 2014 IEEE International Energy Conference (ENERGYCON),. IEEE, 2014, pp. 688-695.

[11] R. Vaze, "Competitive ratio analysis of online algorithms to minimize packet transmission time in energy harvesting communication system," in Proceedings of the IEEE International Conference on Computer Communications, Apr. 2013, pp. 115-1123.

[12] J. Gómez-Vilardebò, "Competitive design of power allocation strategies for energy harvesting wireless communication systems," in Proceedings of the IEEE Global Conference on signal and Information Processing, Dec. 2014, pp. 123-127.

[13] J. Gómez-Vilardebò and D. Gündüz, "Competitive analysis of energy harvesting wireless communication systems," in Proceedings of the IEEE European Wireless Conference, May 2014, pp. 1-6.

[14] E. Hazan, Introduction to Online Convex Optimization, 2015. [Online]. Available: http://ocobook.cs.princeton.edu/

[15] E. Hall and R. Willett, "Online convex optimization in dynamic environments," IEEE J. Sel. Topics Signal Process., vol. 9, no. 4, pp. 647-662, Jun. 2015.

[16] W. Wang and M. A. Carreira-Perpinán, "Projection onto the probability simplex: An efficient algorithm with a simple proof, and an application," arXiv preprint arXiv:1309.1541, 2013.

[17] E. Hazan, A. Agarwal, and S. Kale, "Logarithmic regret algorithms for online convex optimization," Machine Learning, vol. 69, no. 2-3, pp. 169-192, 2007.

[18] S. Boyd and L. Vandenberghe, Convex Optimization. Cambridge Univ Press, 2004 